\title{
3.0-T multiparametric magnetic resonance imaging modifies the template of endoscopic, conventional radical prostatectomy in all cancer risk categories
}

\author{
Mieszko Kozikowski ${ }^{1}$, Jan Powroźnik ${ }^{1}$, Wojciech Malewski ${ }^{1}$, Szymon Kawecki ${ }^{1}$, \\ Sebastian Piotrowicz ${ }^{1}$, Wojciech Michalak ${ }^{1}$, Łukasz Nyk ${ }^{1}$, Magdalena Gola² ${ }^{2}$ Jakub Dobruch ${ }^{1}$
}

\author{
${ }^{1}$ Urology Clinic, Centre of Postgraduate Medical Education, Department of Urology, \\ European Health Centre - Otwock, Poland \\ ${ }^{2}$ Department of Diagnostic Imaging, European Health Centre - Otwock, Poland
}

Submitted: 26 November 2016

Accepted: 23 January 2017

Arch Med Sci 2018; 14, 6: 1387-1393

DOI: https://doi.org/10.5114/aoms.2018.77222

Copyright @ 2018 Termedia \& Banach

\section{Abstract}

Introduction: We aimed to evaluate the diagnostic performance of 3.0-T multiparametric magnetic resonance imaging ( $\mathrm{mpMRI})$ in preoperative staging of prostate cancer $(\mathrm{PCa})$ and its influence on the extent of resection during endoscopic radical prostatectomy (ERP) among cancer risk groups.

Material and methods: The data of 154 patients with PCa in whom mpMRI was performed prior to ERP between 2011 and 2015 were included. The initial decision whether to perform neurovascular bundle (NVB) sparing surgery was based on EAU guidelines. $\mathrm{mpMRI}$ images were reevaluated prior to prostatectomy to modify the surgical template. Imaging was compared with pathological reports to investigate the diagnostic performance of mpMRI.

Results: The surgical template was modified in 69 (44.8\%) patients after reevaluation of mpMRI. More preserving NVB sparing was attempted in $17(11.0 \%)$ men, in whom NVB would have been resected if mpMRI had not been available. More aggressive NVB resection was performed in 52 (33.8\%) men, in whom innervation would have been spared if basing solely based on guidelines. Among all PCa risk groups mpMRI had an impact on the surgical template with more aggressive surgery in $63.0 \%$ and $33.3 \%$ of men in the low- and intermediate-risk group, respectively, and more preserving in $21.4 \%$ of the high-risk patients. The change in extent of resection was not correlated with a higher risk of positive surgical margins $(p=0.196)$.

Conclusions: Preoperative mpMRI exerts a significant impact on decision making concerning the extent of resection during ERP irrespective of the PCa risk group.

Key words: prostate cancer, neurovascular bundle, multiparametric magnetic resonance imaging, extraprostatic extension, endoscopic radical prostatectomy.

\section{Introduction}

Radical prostatectomy (RP) remains the mainstay therapy for patients with localized prostate cancer (PCa) [1]. In comparison to watchful waiting, RP provides significant reduction of cancer specific and all-cause mortality [2]. However, the surgery has been heavily criticized for its ominous influence on quality of life with a third of men suffering from in-

\author{
Corresponding author: \\ Mieszko Kozikowski MD \\ Urology Clinic \\ Medical Centre for \\ Postgraduate Education \\ Department of Urology \\ European Health Centre \\ Borowa 14/18 \\ 05-400 Otwock, Poland \\ Phone: +48 792272890 \\ E-mail: mieszkokozikowski9@ \\ gmail.com
}


continence and more than a half with erectile dysfunction, despite the landmark studies by Walsh et al., which demonstrated the spectacular role of neurovascular bundle (NVB) preservation in retaining potency [3]. Unfortunately, sparing NVBs which lie in very close proximity to the prostate may compromise the pathologic outcome and result in positive surgical margins (PSMs). Positive surgical margins are recognized as an adverse pathologic feature and are known to increase the risk of biochemical recurrence [4]. Therefore, to avoid PSMs, yet preserve the bundles, precise local staging of PCa is of utmost importance. Further, treatment that does not compromise quality of life will become even more important as the incidence of age-related cancers including PCa will rise along with global population aging [5].

Multiparametric magnetic resonance imaging (mpMRI) is currently the most accurate imaging modality that provides clinical data on local extension of PCa. It has been demonstrated that the combination of morphological and functional images offered by modern 3.0-T machines facilitates PCa staging [6]. Although studies with 1.5-T mpMRI confirmed its significant contribution to preoperative planning before robotic assisted laparoscopic radical prostatectomy [7], the European Association of Urology (EAU) recommends implementing mpMRI in the decision-making process in intermediate or high-risk PCa only. As such, EAU does not recognize any additional value of mpMRI in staging of low-risk disease, yet emphasizes the need to attempt NVB-sparing surgery in men with negligible probability of extraprostatic extension (EPE) [1]. In the meantime, 1.5-T mpMRI has been proved in a randomized clinical trial to decrease the rate of PSMs, in low-risk PCa exclusively [8]. Therefore, we sought to determine the value of 3.0-T mpMRI in preoperative planning across all cancer risk categories in patients subjected to conventional, endoscopic radical prostatectomy (ERP).

\section{Material and methods}

\section{Patients}

The data of 154 consecutive patients diagnosed with PCa who underwent conventional ERP preceded by 3.0 Tesla mpMRI between June 2011 and November 2015 were prospectively collected and analyzed. mpMRI was performed for staging purposes in all men prior to ERP but at least 6 weeks after prostate biopsy following ESUR guidelines [6]. The images were reevaluated before surgery to establish the template of resection. Feasibility and extent of NVB-sparing surgery were determined as well. In all cases, the same surgical team responsible for subsequent ERP participated in reevaluation of mpMRI images. The study protocol was approved by the local ethics committee.

\section{Multiparametric magnetic resonance imaging}

mpMRI was performed with an Achieva 3.0-T MRI TX (Philips, Amsterdam, The Netherlands) with dual RF transmitter and 32 independent receiving channels. The MRI protocol included: axial T2-weighted turbo spin echo sequence, axial diffusion-weighted imaging spin echo sequence with apparent diffusion coefficient map, axial dynamic contrast-enhanced imaging, axial T1-weighted spin echo with selective fat suppression sequence, axial T1-weighted turbo field echo sequence, and coronal and sagittal T2-weighted turbo spin echo sequence in all cases. The images were interpreted by a single experienced radiologist specialized in genitourinary tract diagnostics, who was not blinded to clinical metrics including serum prostate-specific antigen (PSA), cTNM or biopsy results. Irregular shape of prostatic capsule, seminal vesicle invasion, periprostatic fat infiltration, bladder neck invasion and infiltration of rectum wall were all acknowledged to reflect the presence of EPE. Finally, mpMRI was reevaluated by the surgical team in cooperation with the radiologist just before ERP to individualize the approach with respect to the surgical template that involved NVB preservation.

\section{Endoscopic radical prostatectomy}

Endoscopic radical prostatectomy was carried out by the same surgeons having significant experience in oncological, endoscopic surgery. The initial surgical template was established according to EAU recommendations based on all clinical features including transrectal ultrasound (TRUS), digital rectal examination (DRE), PSA, Gleason score and tumor extent in prostate biopsy except mpMRI [1]. In low-risk cases (PCa cT $\leq 1 \mathrm{a}, \mathrm{GS}<7$ in biopsy and PSA below $10 \mathrm{ng} / \mathrm{ml}$ ) extraperitoneal ERP was performed. Otherwise patients underwent transperitoneal ERP with extended pelvic lymph node dissection [9]. The decision concerning the extent of NVB preservation was made in an individual manner based on clinical characteristics. Following EAU guidelines 2015, in case of PCa $\mathrm{CT} \geq 2$, any biopsy with GS $>7$ or more than one biopsy core with GS > 6 at the ipsilateral side with PSA above $10 \mathrm{ng} / \mathrm{ml}$, NVB removal was planned [9]. The extent of resection remained unchanged unless suggested otherwise by the mpMRI images presenting the distant location of the PCa lesion in relation to neurovascular structures. Both radiologist and urologists were involved in the decision-making process in regard to the dissection plan. It was labeled unchanged if not modified 
after reevaluation of mpMRI images. Otherwise the surgical template was considered as changed toward either a more preserving NVB-sparing approach or more aggressive NVB resection.

The bundles were preserved separately on both sides with either an interfascial or an intrafascial approach. Intrafascial technique reflected the widest template of NVB sparing, while the interfascial approach was associated with partial removal of neurovascular structures. In cases of negative mpMRI and negative biopsy NVB preservation was attempted: intrafascially on that side if low-risk cancer was detected on the contralateral side; interfascially on that side if intermediate to high-risk cancer was detected on the contralateral side. No preservation was implemented if mpMRI suggested EPE or tumor focus adjacent to NVB on the ipsilateral side.

\section{Histopathological examination}

The specimens were examined by a pathologist devoted to oncological urology. In case of adverse pathology the report was reevaluated by another experienced uropathologist together with urologists. The histopathological report always included at least prostate volume, type of cancer, Gleason score, tumor location and extent, and surgical margin status. According to the EAU guidelines EPE was defined as the presence of tumor beyond the prostate capsule and was substaged into extracapsular extension and seminal vesicle invasion. For the purpose of our analysis the study group was divided into localized ( $\leq \mathrm{T} 2 \mathrm{c}$ ) and locally advanced disease ( $\geq T 3 a$ ) based on mpMRI and the histopathology report. Surgical margins were positive if cancer cells were found within the inked surface of the prostate specimen. Their location was scrutinized and registered on the prostate map to see whether the modification of the surgical template after mpMRI reevaluation was responsible for the PSMs.

\section{Statistical analysis}

The epiR and pROC packages of the program $R$ (version 3.0.2, the $R$ foundation for Statistical Computing, www.r-project.org) were used to perform statistical analysis. Descriptive statistics are reported as mean \pm standard deviation. Diagnostic performance was expressed with sensitivity, specificity, positive and negative predictive values and accuracy with $95 \%$ confidence interval. The histopathological report was regarded as a reference standard.

$\chi^{2}$ test and Fisher's exact test were performed when appropriate. The significance level was set to $p<0.05$. The DeLong test was used to compare area under the curve (AUC) in subgroup analysis to reveal differences in the diagnostic performance.

\section{Results}

\section{Clinical characteristics}

Baseline clinical characteristics of all 154 men included in the study are presented in Table I. There were 27 (17.5\%), 57 (37.0\%) and 70 (45.5\%) cases classified into low, intermediate or high-risk EAU categories, respectively. Prior to $\mathrm{mpMRI}$ and according to EAU recommendations, unilateral and bilateral NVB-sparing surgery was anticipated in 51 (33.1\%) and 59 (38.3\%) cases, accordingly. Forty-four (28.6\%) patients would be disqualified from any type of NVB-sparing surgery.

\section{Magnetic resonance imaging performance}

mpMRI suggested EPE in 27 (17.5\%) males, whereas in the remaining 127 (82.5\%) cases PCa was either confined to the prostate or no $a b$ normal lesion was identified in 114 (74.0\%) and

Table I. Clinical characteristics of 154 men included in the study

\begin{tabular}{|c|c|}
\hline Parameter & Value \\
\hline Age, mean \pm SD [years] & $62.5 \pm 7.0$ \\
\hline $\mathrm{PSA}$, mean $\pm \mathrm{SD}[\mathrm{ng} / \mathrm{ml}]$ & $10.6 \pm 11.4$ \\
\hline Prostate volume, mean \pm SD $[\mathrm{ml}]$ & $42.7 \pm 19.7$ \\
\hline $\mathrm{PSAD}$, mean $\pm \mathrm{SD}\left[\mathrm{ng} / \mathrm{ml}^{2}\right]$ & $0.29 \pm 0.33$ \\
\hline \multicolumn{2}{|l|}{ GS in biopsy: } \\
\hline$\leq 6$ & $56(36.3 \%)$ \\
\hline 7 & $74(48.1 \%)$ \\
\hline$\geq 8$ & $24(15.6 \%)$ \\
\hline \multicolumn{2}{|l|}{ cTNM: } \\
\hline$\leq \mathrm{cT} 2$ & $127(82.5 \%)$ \\
\hline$\geq \mathrm{cT3}$ & $27(17.5 \%)$ \\
\hline \multicolumn{2}{|l|}{ GS in histopathology: } \\
\hline$\leq 6$ & $40(26.0 \%)$ \\
\hline 7 & $86(55.8 \%)$ \\
\hline$\geq 8$ & $28(18.2 \%)$ \\
\hline \multicolumn{2}{|l|}{ pTNM: } \\
\hline$\leq \mathrm{pT} 2$ & $105(68.2 \%)$ \\
\hline$\geq \mathrm{pT3}$ & $49(31.8 \%)$ \\
\hline \multicolumn{2}{|l|}{ Surgical margin status: } \\
\hline PSM- & $131(85.1 \%)$ \\
\hline PSM+ & $23(14.9 \%)$ \\
\hline
\end{tabular}

SD - standard deviation, PSA - prostate specific antigen, PSAD - prostate specific antigen density, GS - Gleason score, PSM positive surgical margin. 
$13(8.5 \%)$ patients, respectively. The stage of PCa determined due to $\mathrm{mpMRI}$ was consistent with pathological stage in $118(76.6 \%)$ men. Understaging was revealed in 29 (18.8\%) patients, whereas overstaging occurred in 7 (4.5\%) males only. In the entire cohort, sensitivity, specificity, positive and negative predictive values and accuracy of $\mathrm{mpMRI}$ in EPE detection were $41 \%, 93 \%, 74 \%, 77 \%$ and $76 \%$, respectively.

Despite differences in crude numbers, comparison of AUC revealed that mpMRI diagnostic performance does not differ significantly between particular risk categories $(p=0.123$ ). Sensitivity, specificity, positive and negative predictive values and accuracy were $20 \%, 96 \%, 50 \%, 85 \%, 82 \%$ in the combined low to intermediate-risk group and $50 \%, 89 \%, 81 \%, 65 \%, 70 \%$ in the high-risk group, respectively.

\section{Surgical plan}

The flowchart of men initially subjected to bilateral, unilateral or no NVB preservation and modification of this plan based on the mpMRI result among the particular risk groups is illustrated in Figure 1. After mpMRI reevaluation, NVBs were spared on both sides or on one side in 32 (20.8\%) and in 78 (50.6\%) men respectively. They were not preserved in $44(28.6 \%)$ cases. The extent of NVB-sparing surgery was modified in 69 (44.8\%) men. In the low and intermediate-risk group the decision after mpMRI reevaluation was mainly changed towards more aggressive resection, whereas in the high-risk group the extent of NVB sparing was modified in both directions (Figure 1).

In $17(11.0 \%)$ cases the extent of NVB preservation was changed towards a more preserving approach: on both sides in 4/17 (23.5\%) men and on one side in 13/17 men (76.5\%). In 52 (33.8\%) cases the surgery was more aggressive and the NVB-sparing approach was narrowed to an interfascial bilateral or unilateral procedure in 6/52 (11.5\%) and in 31/52 (59.6\%) males accordingly, whereas in 15/52 (28.9\%) men bundles were removed completely on both sides.

\section{Pathological outcome}

The overall PSM rate was $14.9 \%(n=23)$. PSM was revealed in $17 / 49$ (34.7\%) men with EPE and it was significantly more prevalent $(p<0.01)$ than in those with organ confined disease, of which 6/105 (5.7\%) had PSM. Positive margins were found in $6(8.2 \%)$ men with unilateral and $2(6.3 \%)$ men after bilateral NVB-sparing surgery and $16(32.7 \%)$ men with NVB removal.

The risk of leaving PSMs did not differ significantly irrespective of the decision made after the $\mathrm{mpMRI}$ reevaluation (Table II). In patients in whom the initial decision was modified towards a more preserving approach, positive margins were found in $3 / 17(17.6 \%)$ cases, whereas in those in whom the resection was more aggressive PSMs were observed in 4/52 (7.7\%) males. However, in only one case PSM was found at the site where the decision was changed to the more preserving NVB surgery. Despite the decision of more aggressive resection, three-quarters of these men had PSM on that side.

\section{Discussion}

Our study provides critical insight into the decision-making process that concerns the extent of resection during ERP. We found that preoperative $\mathrm{mPMRI}$ influenced the approach towards

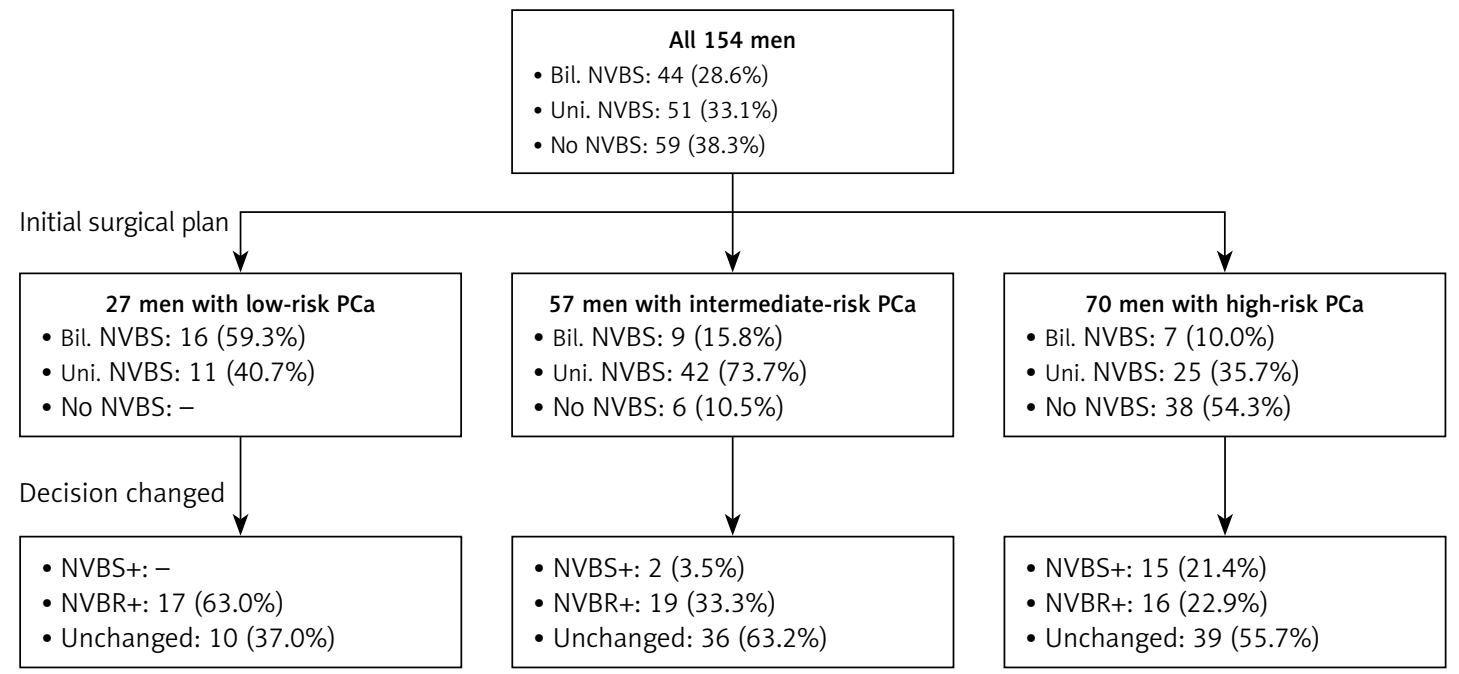

Figure 1. The initial surgical plan about NVB preservation and the change in decision based on the mpMRI result Bil. - bilateral, Uni. - unilateral, NVBS+-more preserving neurovascular bundle sparing, NVBR+-more aggressive neurovascular bundle resection, $P C a$ - prostate cancer, $m p M R I$ - multiparametric magnetic resonance imaging. 
Table II. The modification of surgical template related to EPE detection in mpMRI

\begin{tabular}{|lcccccc|}
\hline $\begin{array}{l}\text { Decision about NVB } \\
\text { preservation }\end{array}$ & $\begin{array}{c}\text { Unchanged } \\
\text { decision about } \\
\text { NVB sparing } \\
\text { Group A }(n=85)\end{array}$ & $\begin{array}{c}\text { More preserving } \\
\text { NVB sparing } \\
\text { Group B }(n=17)\end{array}$ & $\begin{array}{c}\text { More aggressive } \\
\text { NVB resection } \\
\text { Group C }(n=52)\end{array}$ & $\begin{array}{c}P \text {-value } \\
\text { A vs. B }\end{array}$ & $\begin{array}{c}P \text {-value } \\
\text { A vs. C }\end{array}$ & $\begin{array}{c}\text { Overall } \\
p \text {-value }\end{array}$ \\
\hline \begin{tabular}{l} 
mpMRI result: \\
\hline EPE-
\end{tabular} & $64(75.3 \%)$ & $16(94.1 \%)$ & $47(90.4 \%)$ & 0.111 & $0.049^{*}$ & $0.038^{*}$ \\
\hline EPE+ & $21(24.3 \%)$ & $1(5.9 \%)$ & $5(9.6 \%)$ & & & \\
\hline PTNM: & $51(60.0 \%)$ & $11(64.7 \%)$ & $43(82.7 \%)$ & 0.928 & $0.010^{*}$ & $0.021^{*}$ \\
\hline$\leq$ PT2 & $34(40.0 \%)$ & $6(35.3 \%)$ & $9(17.3 \%)$ & & & \\
\hline$\geq P T 3$ & $69(81.2 \%)$ & $14(82.4 \%)$ & $48(92.3 \%)$ & 1.000 & 0.085 & 0.196 \\
\hline PSM: & $16(18.8 \%)$ & $3(17.6 \%)$ & $4(7.7 \%)$ & & & \\
\hline PSM- & & & & & \\
\hline PSM+ & & & & & & \\
\hline
\end{tabular}

$N V B$ - neurovascular bundle, EPE - extraprostatic extension, mpMRI - multiparametric magnetic resonance imaging, NVB - neuromuscular bundle, PSM - positive surgical margin. ${ }^{*} P<0.05$.

NVB regardless of $P C a$ risk category. Men from all $\mathrm{PCa}$ risk groups benefit from the preoperative mpMRI, but these advantages vary among the groups depending on the particular clinical scenario. Of note, modification of the initial surgical plan by mpMRI does not increase the risk of PSMs.

The NVB preservation is associated with recovery of erectile function after RP [10]. Moreover, increasing evidence suggests that bundle-sparing surgery may have an impact on urinary continence, though more robust data are needed to confirm this observation [11]. Although no single study has definitely proved that nerve sparing improves continence, an association between the extent of resection during RP and continence has already been acknowledged [12]. Therefore, an attempt to spare NVB should be made whenever possible. Recent improvements in surgical techniques ended the era of a binary approach towards NVB sparing and the introduction of mpMRI as a novel diagnostic tool providing visual feedback on tumor location and extent made the preoperative planning more flexible [13]. In our report, mpMRI led to significant modification of the surgical template in almost half $(44.8 \%)$ of the cases. Although this rate varies among different studies, it indicates a great influence of MRI on the decision-making process prior to $\operatorname{RP}[7,8,14]$. Moreover, it has been reported that MRI has a substantial impact on the extent of resection in other surgical techniques such as robotic-assisted laparoscopic RP and open RP as well $[7,14]$. While we have noted, like other authors, a tendency toward more aggressive NVB resection after MRI reevaluation (33.8\%), we also found that in selected cases (11.0\%) the decision may be opposite, strengthening more preserving surgery $[8$, 14]. This trend is particularly established in the high-risk group with $21.4 \%$ of nerve-sparing surgery performed despite initial contraindications. The decision in such cases was mainly driven by a favorable tumor location within the prostate gland limits. It has been demonstrated that mpMRI has reasonably high negative predictive value in excluding EPE in the high-risk group. As an example, more preserving NVB sparing is feasible when PCa lesions are located within the central zone.

\section{mpMRI performance}

Combination of high spatial resolution T2-weighted imaging with functional mpMRI sequences has become the mainstay staging modality in PCa [6]. In our study 3.0-T MRI technology that embraced T2-weighted sequences with other functional mpMRI images was implemented for staging purposes. The sensitivity and specificity of such an approach in the detection of EPE were $40 \%$ and $93 \%$ respectively. According to recently published meta-analysis, corresponding values ranged from $54 \%$ to $67 \%$ and from $85 \%$ to $91 \%$, accordingly [15]. The variation reflects the differences in MRI machines, diverse attitudes of radiologists to the signs considered indicative of EPE as well as disparate histopathological approaches to extracapsular extension across the studies. Furthermore, the sensitivity of mpMRI increases significantly when extensive, but not focal, EPE is identified [16]. The low sensitivity in our study may be explained by a definite criteria adopted by our radiologist, who reported EPE in cases when direct signs of extraprostatic disease are present, which in turn resulted in high specificity. 


\section{$\mathrm{mpMRI}$ in prostate cancer risk categories}

Operating surgeons are less likely to spare the bundles when MRI indicates locally advanced disease $[17,18]$. However, we did not see such a tendency in our study, having a relatively low level of EPE suspicion in the group, in which the decision was changed to more aggressive NVB resection (Table II). This phenomenon underscores the multifactorial aspects of preoperative planning in PCa. Patients' expectations and surgeons' receptivity have to be acknowledged. More aggressive NVB resection was driven by unfavorable tumor location and inherent possibility of EPE. Even in cases of small lesions, yet located in the peripheral zones close to the bundles, the operating team decided to expand the resection and incorporate interfascial instead of intrafascial NVB-sparing technique. This approach is reflected by the rate of $33.8 \%$ of more aggressive resection, despite the possibility of NVB preservation according to EAU guidelines. Not surprisingly, the decision to broaden the template was most commonly made in the lowrisk PCa group (63.0\%), despite low sensitivity of mpMRI in EPE detection. Our results corroborate the suggestions presented by other authors that mpMRI provides clinically important data in T1c cases [8]. Simultaneously, the reports indicating minimal value of $M R I$ in the decision to change the surgical approach underline that MRI provides reassurance in excluding EPE in men who undergo NVB-sparing surgery [14]. Presumably mpMRI improves clinical staging in these patients by providing visual information about tumor extent, therefore preventing intrafascial NVB sparing.

EAU guidelines recommend mpMRI for local staging in high-risk PCa patients. Our study revealed that sensitivity (49\%) was better among men with high-risk PCa, which is consistent with other reports, where subgroup analysis was performed and mpMRI sensitivity in EPE detection increased in concordance with the EAU risk group [17-19]. mpMRI showed relatively high positive predictive value (81\%) in detecting EPE; therefore men with high-risk PCa and mpMRI suggesting EPE should be disqualified from NVB-sparing surgery at least on the affected side.

The low- to intermediate-risk group is less likely to benefit from MRI preoperative staging, although the moderate negative predictive value (85\%) suggests that NVB-sparing surgery may be performed when mpMRI reveals no signs of EPE. The results suggest that mpMRI is a useful tool to discriminate between localized and locally advanced disease before surgery, especially in the high-risk group. In summary, mpMRI provides clinically useful information in every PCa risk category. As such, it is usually followed by more aggres- sive surgery in low-risk PCa patients and a more restrictive approach in high-risk PCa patients.

\section{Positive surgical margins rate}

NVB-sparing surgery cannot compromise the completeness of cancer excision. In our study, modification of the surgical template was not associated with higher risk of PSMs ( $p=0.133)$. Other studies have also found that decisions on NVB preservation made after MRI had no influence on PSM rates [7, 20]. However, in one study, in cases in which MRI erroneously excluded EPE, it led to more preserving NVB surgery which resulted in an increased PSM rate after laparoscopic RP [21]. The effect was most striking in low-risk tumors, in which an $80 \%$ PSM rate was observed [21]. We did not observe such a relationship in our study. On the contrary, the majority of PSMs were found in the high-risk group (21 cases, 87.5\%) and the mpMRI modification of the surgical plan did not correlate with a higher risk of PSMs ( $p=$ 0.07). Furthermore, in the subgroup of men with more preserving surgery PSMs were usually found at a site that was located far from the region of bundle sparing. Conversely, there were some PSM cases identified in spite of more aggressive NVB resection at that site. This reveals a shortcoming of the presented approach incorporating mpMRI in surgical planning among cases with locally advanced disease in which PSMs seem unavoidable, despite correct imaging. The results of a recently published randomized clinical trial showed no reduction of the overall risk of PSM in high-risk PCa patients, who had MRI done prior to RP [8]. However, in men with T1c tumors there was an absolute reduction of PSM rate by $11 \%$, indicating that at least selected patients benefit from preoperative $\mathrm{MRI}$ in regard to the surgical margin status. Of note, mpMRI sensitivity in EPE detection may not be decisive in preventing PSM, as it is not rare for PSM to occur in different locations than EPE [22]. Nevertheless, NVB-sparing surgery is not advisable when EPE is suspected on mpMRI. One innovative technique, which gives promising results in reducing the PSM rate and is also recommended as an auxiliary method by the EAU, is intraoperative frozen-section analysis directed at the lesions detected in MRI. However, to the best of our knowledge, the data on use of frozen-section come from several retrospective studies and therefore should be validated in randomized controlled trials with longer follow-up [23].

We acknowledge several limitations of our study. The 3.0-T mpMRI protocol was not uniform for all patients, as in several cases magnetic resonance spectroscopic imaging was additionally performed. The radiologist was not blinded to the clinical characteristics of our patients, which 
could influence the interpretation of mpMRI images. However, it reflects daily clinical practice. Although baseline erectile function and continence were recorded as part of the standard preoperative workup, we did not provide any further analysis of functional outcomes after surgery. Further prospective studies including more centers are required to determine which patients would benefit the most from mpMRI preoperative planning.

In conclusion, preoperative mpMRI providing accurate information on tumor location and extent significantly influences the decision on the extent of resection during ERP irrespective of the $\mathrm{PCa}$ risk category. Template modification driven by $\mathrm{mpMRI}$ to perform more preserving or more aggressive surgery with respect to NVB does not portend higher risk of PSMs. Close cooperation between radiologists and urologists is necessary to provide an optimal outcome. Our observations strongly support incorporation of $\mathrm{mpMRI}$ into the preoperative workup of patients selected for RP.

\section{Conflict of interest}

The authors declare no conflict of interest.

\section{References}

1. Heidenreich A, Bastian PJ, Bellmunt J, et al. EAU guidelines on prostate cancer. part 1: screening, diagnosis, and local treatment with curative intent-update 2013. Eur Urol 2014; 65: 124-37.

2. Bill-Axelson A, Holmberg L, Filen F, et al. Radical prostatectomy versus watchful waiting in localized prostate cancer: the Scandinavian prostate cancer group-4 randomized trial. J Natl Cancer Inst 2008; 100: 1144-54.

3. Quinlan DM, Epstein JI, Carter BS, Walsh PC. Sexual function following radical prostatectomy: influence of preservation of neurovascular bundles. J Urol 1991; 145: 998-1002.

4. Yossepowitch O, Briganti A, Eastham JA, et al. Positive surgical margins after radical prostatectomy: a systematic review and contemporary update. Eur Urol 2014; 65: 303-13.

5. Golabek T, Powroźnik J, Chłosta P, et al. The impact of nutrition in urogenital cancers. Arch Med Sci 2015; 11: 411-8.

6. Barentsz JO, Richenberg J, Clements R, et al. ESUR prostate MR guidelines 2012. Eur Radiol 2012; 22: 746-57.

7. Park BH, Jeon $H G$, Jeong $B C$, et al. Influence of magnetic resonance imaging in the decision to preserve or resect neurovascular bundles at robotic assisted laparoscopic radical prostatectomy. J Urol 2014; 192: 82-8.

8. Rud E, Baco E, Klotz D, et al. Does preoperative magnetic resonance imaging reduce the rate of positive surgical margins at radical prostatectomy in a randomised clinical trial? Eur Urol 2015; 68: 487-96.

9. Mottet N, Bellmunt J, et al. EAU guidelines on prostate cancer: 6.2.10 Indications for nerve-sparing surgery. European Association of Urology 2015. Available at: https://uroweb.org/guideline/prostate-cancer/.

10. Salonia A, Burnett AL, Graefen M, et al. Prevention and management of postprostatectomy sexual dysfunctions.
Part 1: choosing the right patient at the right time for the right surgery. Eur Urol 2012; 62: 261-72.

11. Steineck G, Bjartell A, Hugosson J, et al. Degree of preservation of the neurovascular bundles during radical prostatectomy and urinary continence 1 year after surgery. Eur Urol 2015; 67: 559-68.

12. Michl U, Tennstedt P, Feldmeier L, et al. Nerve-sparing surgery technique, not the preservation of the neurovascular bundles, leads to improved long-term continence rates after radical prostatectomy. Eur Urol 2016; 69: 584-9.

13. Tan N, Margolis DJ, McClure TD, et al. Radical prostatectomy: value of prostate MRI in surgical planning. Abdom Imaging 2012; 37: 664-74.

14. Hricak H, Wang L, Wei DC, et al. The role of preoperative endorectal magnetic resonance imaging in the decision regarding whether to preserve or resect neurovascular bundles during radical retropubic prostatectomy. Cancer 2004; 100: 2655-63.

15. de Rooij M, Hamoen EH, Witjes JA, Barentsz JO, Rovers MM. Accuracy of magnetic resonance imaging for local staging of prostate cancer: a diagnostic meta-analysis. Eur Urol 2016; 70: 233-45.

16. Feng TS, Sharif-Afshar AR, Smith SC, et al. Multiparametric magnetic resonance imaging localizes established extracapsular extension of prostate cancer. Urol Oncol 2015; 33: 109 e15-22.

17. Roethke MC, Lichy MP, Kniess M, et al. Accuracy of preoperative endorectal MRI in predicting extracapsular extension and influence on neurovascular bundle sparing in radical prostatectomy. World J Urol 2013; 31: 1111-6.

18. Tanaka K, Shigemura K, Muramaki M, Takahashi S, Miyake $\mathrm{H}$, Fujisawa M. Efficacy of using three-tesla magnetic resonance imaging diagnosis of capsule invasion for decision-making about neurovascular bundle preservation in robotic-assisted radical prostatectomy. Korean J Urol 2013; 54: 437-41.

19. Rud E, Klotz D, Rennesund K, et al. Preoperative magnetic resonance imaging for detecting uni- and bilateral extraprostatic disease in patients with prostate cancer. World J Urol 2015; 33: 1015-21.

20. McClure TD, Margolis DJ, Reiter RE, et al. Use of MR imaging to determine preservation of the neurovascular bundles at robotic-assisted laparoscopic prostatectomy. Radiology 2012; 262: 874-83.

21. Brown JA, Rodin DM, Harisinghani M, Dahl DM. Impact of preoperative endorectal MRI stage classification on neurovascular bundle sparing aggressiveness and the radical prostatectomy positive margin rate. Urol Oncol 2009; 27: 174-9.

22. Johnson MT, Ramsey ML, Ebel JJ, Abaza R, Zynger DL. Do robotic prostatectomy positive surgical margins occur in the same location as extraprostatic extension? World J Urol 2014; 32: 761-7.

23. Petralia G, Musi G, Padhani AR, et al. Robot-assisted radical prostatectomy: Multiparametric MR imaging-directed intraoperative frozen-section analysis to reduce the rate of positive surgical margins. Radiology 2015; 274: 434-44. 